\title{
An indigenously developed electronic control system for Langmuir-Blodgett film deposition set-up
}

\author{
C P NAVATHE, B L DASHORA, ARCHANA JAISWAL ${ }^{a}$, \\ D S THAKUR, U N ROY and L M KUKREJA
}

Laser Programme, Centre for Advanced Technology, Indore 452 013, India ${ }^{a}$ Present address: Inter University Consortium for DAE Facilities, Indore 452 017, India

e-mail: cpn@cat.ernet.in

MS received 23 November 2000; revised 9 March 2001

\begin{abstract}
An indigenous and simple electronic control system for Langmuir-Blodgett (LB) film deposition set-up has been developed. This set-up consists of a microstepping circuit to drive the stepper motors with precision and smooth motion, essential for controlled movement of the barriers and substrate in the LB set-up. Linear variable differential transformer (LVDT)-based displacement measuring device has been developed and used to measure the surface pressure of the monolayer material spread on the water surface. A control program is written which incorporates all operational modes required to drive the set-up and to acquire the data in situ using a set of user-friendly commands. This control set-up has been successfully used to plot the pressure-area isotherm of various amphiphilic compounds such as ferric stearate, zinc arachidate etc. and for deposition of ordered LB films of ferric stearate.
\end{abstract}

Keywords. Electronic control system, Langmuir-Blodgett set-up, microstepping.

\section{Introduction}

The study of thin organic films has expanded enormously in recent years due to their potential application as useful components in many practical and commercial devices such as sensors, detectors, displays, electronic circuits etc. (Benming 1991; Ulman 1991; Casy \& McMonagle 1996). The high degrees of order in Langmuir-Blodgett (LB) films could lead to systems in which the molecules themselves become the active electronic components. The possibility of developing molecular level electronics has fascinated many scientists and given rise to attempts towards constructing organised 
systems of co-operating molecules, i.e. systems of molecules that interlock and interact like parts of a machine (Peterson 1992). Many efforts are being made to assemble the molecules in a well-ordered way, using a variety of different deposition techniques (Kuhn et al 1972; Tredgold 1994). The Langmuir-Blodgett (LB) technique is one of the most promising techniques for preparing such thin films as it enables the fabrication of highly ordered monlayers and multilayers of controllable thickness at the molecular level (Hann 1991; Bryce \& Petty 1995; Petty 1996).

Design and fabrication of the set-up for deposition of LB films have to be carried out taking into account the following aspects: High level of cleanliness; very fine and smooth movement of the mechanical parts; accurate and precise measurements and data recording of different parameters before and/or during the growth of the films. Since these are highly ordered ultrathin films of layer thicknesses of a few Angstroms, negligence in any of the above mentioned aspects while designing the set-up would lead to serious difficulties in getting good quality films. The electronic system of the LB set-up, which controls the movements of all mechanical parts, measurements and data recording is, therefore, quite complicated and needs great attention in order to fulfil the above requirements.

The usual LB set-up consists of a trough containing a subphase, normally water, on which the molecules (essentially the surface active molecules) to be deposited as LB film are spread. The surface area of the trough can be varied by sweeping with movable barriers over the surface of the trough. Sweeping with a barrier over the water surface causes the molecules to come close together and eventually to form a compressed ordered monolayer. Progress of the formation of film and its properties are studied by continuously monitoring the surface pressure during compression.

Surface pressure is measured by the Wilhelmy-plate method. In this method, the measurement is made by determining the force due to surface tension on a plate partially immersed in the subphase. This force is then converted into surface tension $(\mathrm{mN} / \mathrm{m})$ using the dimensions of the plate. The floating compressed monolayer is deposited onto a solid substrate by moving it up and down through the monolayer while simultaneously keeping the surface pressure constant with a computer-controlled feedback system between the Wilhelmy plate measuring the surface pressure and the barrier-moving mechanism. The motion of the barrier as well as the substrate should be absolutely vibration-free in order to get uniform films. The control of the barrier and the substrate motion in the LB set-up is also carried out by the computer-based system and a number of reports are available in the literature on this aspect (Miller et al 1988; Albrecht 1983; Peterson 1985; Brockman et al 1980).

In this paper we describe a microprocessor-based control system for the LB set-up indigenously developed by us. This system is simpler, modular and more economic than the commercial systems. At the same time, it provides high accuracy and reliability of performance, as required.

\section{Description of the overall system}

Figure 1 represents the block diagram of the experimental LB set-up developed at our laboratory. The LB trough is rectangular in shape and is made of a polymeric material commonly known as perspex. The barriers are made of Teflon and are sufficiently 
smooth so that they can slide easily on the walls of the trough. They are driven by a single lead screw which had threads of opposite rotation on two sides. Thus when it is rotated in one direction, both barriers move inwards; and when it is rotated in opposite direction, barriers move outwards. The substrate is hung by means of a pulley and thread to the second motor and it slides up and down through two guides. The Wilhelmy plate is attached by a thread to the core of LVDT, and this core in turn is attached to a spring made of phosphor bronze. The Wilhelmy plate can be shifted up and down by an adjustable arrangement. The whole assembly of the trough is kept on vibration-free supports made using several slabs of thermocole sheets, so that vibrations caused due to nearby objects are not transferred to the deposition set-up. The unit is enclosed in a cabinet with the front made of glass, so that the film is not disturbed by air currents but the process of deposition can be observed by the operator.

The electrical system can be divided into the following sub systems.

(i) Horizontal movement of barriers and vertical movement of substrate: This motion can be given either with DC motors or stepper motors. DC servomotors are generally preferred for this purpose due to the smoothness of the motion required. But use of DC motors leads to further complexity in the system due to following reasons. In DC motors, speed of rotation is proportional to applied voltage, so they run continuously at some particular speed at applied voltage. Therefore it is necessary to use transducers for measuring the displacement. Also, the speed vs. voltage characteristic of the motor is valid over limited range, which has to be taken into account while designing the system.

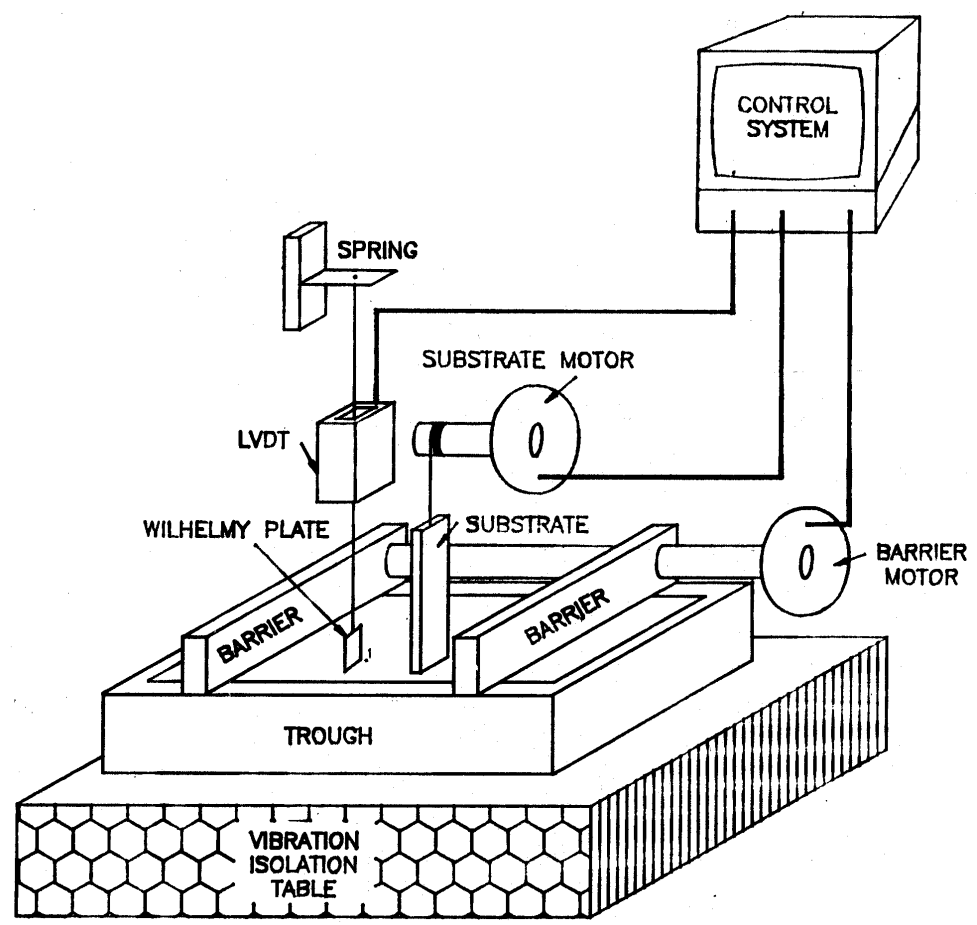

Figure 1. Block diagram of the LB film deposition set-up. 
On the contrary, stepper motors can be conveniently used to generate precise controlled motion, but as their motion is in steps, it is jerky. In recent times, it has been found that stepper motors can be made to run in smooth manner by operating them in microstepping model (Ciscato et al 1988; Wynne 1990). This method enables one to run the motor at slow speeds in a smooth manner and also increases the overall resolution of the step size. Hence we have used stepper motors for moving the barriers and the substrate (Navathe et al 1998).

(ii) Measurement of surface pressure: This is done with the help of a Wilhelmy plate. As the monolayer is compressed or expanded, the Wilhelmy plate moves out of or into the subphase. For detection of such motion, a highly sensitive detector with high resolution is required, hence we have chosen a linear variable differential transformer (LVDT) for this purpose.

(iii) A microcomputer: A microcomputer is necessary for interfacing different motion controllers and measurement circuits included in this set-up and for operating the set-up in various modes as per the requirements of the user. We have used a microprocessor for carrying out the low level jobs of data acquisition and control of motors. The microcomputer is connected through a serial port of the personal computer (PC), which provides an interface to the user. Various operations required for the control of the LB set-up are carried out by the control program developed on the PC.

Each of these subsystems is described in detail in following subsections.

\subsection{Stepper motor controller}

The horizontal motion of the barriers and the vertical motion of the substrate is controlled by identical stepper motors and therefore the stepper motor driver circuits for both are also similar.

A stepper motor consists of windings divided in two or four phases. These windings are driven by a square waveform, which can be generated by digital control logic, driving power transistors. However, for getting smooth motion as well as for increasing its resolution, this waveform is modified to sinusoidal. As a result, the driving circuit has to generate four sinusoidal waveforms, in proper phase sequence and also capable of driving the motor load.

The stepper motor used in our application has two windings which require bipolar current drive. It has a resistance of $40 \Omega$ per phase, resolution of 200 steps per revolution, and maximum speed of 200 steps/second. For driving this motor in microstepping mode, we have generated sinusoidal waveforms with the help of four D/A converters. A parallel output port PA of I/O chip 8255 is used to load data in four $\mathrm{D} / \mathrm{A}$ converters, AD 7524. The data load pulses are generated from I/O lines of port PB. The signal level is changed to bipolar waveform with peak-to-peak amplitude of 12 volts and applied through current amplifier. The block diagram of this circuit is shown in figure 2 .

By applying proper digital data to the inputs of D/A converters, sinusoidal waveforms with phase difference of $90^{\circ}$ are generated, which are used to drive two phases of the motor. Using 8-bit words, 64 levels are defined to generate the sinusoidal waveform. One cycle of the sine wave is therefore generated in 128 steps. Thus, for every 4 macrosteps, which are generated due to square wave, 128 microsteps are 
generated, i.e. 1 macrostep is divided into 32 microsteps. As the motor completes one revolution in 200 steps, the total number of steps per revolution becomes 6400 .

The resolution of substrate motion is $2.34 \mu \mathrm{m} / \mathrm{step}$ and maximum speed is $1.17 \mathrm{~mm} / \mathrm{s}$, in our set-up, whereas for barriers, resolution in $0.29 \mu \mathrm{m} / \mathrm{step}$ and maximum speed is $0.145 \mathrm{~mm} / \mathrm{s}$.

\subsection{LVDT interface}

Surface pressure of the monolayer is measured with the help of Wilhelmy plate as mentioned earlier. The maximum displacement due to this is of the order of $2 \mathrm{~mm}$, and has to be measured with a resolution of $10 \mu \mathrm{m}$. The LVDT and its interface circuit are described as follows.

An LVDT consists of a one primary winding and two secondary windings. The core moves freely inside, so as to keep friction at the minimum level.

The primary is excited by the sinusoidal source and the primary current is $i p$. Then the voltage induced in two secondaries is given as:

$$
\begin{aligned}
& e s 1=M 1 \times \mathrm{dip} / \mathrm{d} t, \\
& e s 2=M 2 \times \mathrm{dip} / \mathrm{d} t,
\end{aligned}
$$

where $M 1$ and $M 2$ are their respective mutual inductances. When the core is at the centre, symmetrical with respect to both secondaries, $M 1=M 2$ and es $1=e s 2$. As the core moves in either direction, net mutual inductance $(M 1-M 2)$ varies linearly. As a result, the difference (between es 1 and es2) varies linearly, and can be used to detect the displacement.

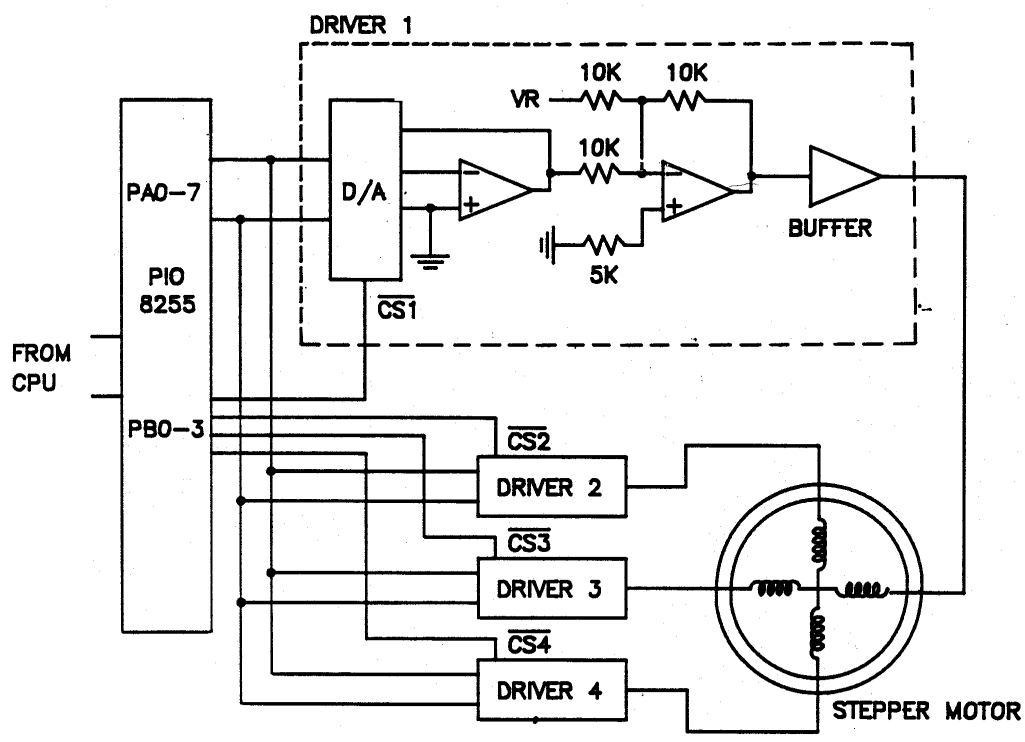

Figure 2. Stepper motor driver circuit. 
The LVDT developed for this application is wound with the primary and two secondary coils of 1000 turns each. The choice of core was made on the basis of its light weight, as the Wilhelmy plate can drive only very light loads. The core selected for this purpose was I section of the ferrite core. This core was cut longitudinally to reduce its weight further.

2.2a Input drive for LVDT: The input signal driving the primary coil has to be of stable frequency and amplitude. Hence a crystal oscillator operating at $4.9152 \mathrm{MHz}$ is used to generate a square wave. This square wave is used to address a ROM by means of a binary counter. The data stored in the ROM is applied to a 8-bit D/A converter. As the ROM contains the digital equivalent of a sine wave, the D/A converter produces sinusoidal waveforms. The frequency of this signal can be adjusted in steps by varying the divider setting. Actual frequency used in our case is $9.6 \mathrm{kHz}$. This waveform is passed through a low pass filter and applied to the primary of LVDT. This method ensures that the frequency as well as the amplitude of the drive signal area stable.

2.2b Output signal conditioning: The LVDT has two secondary output coils. It is necessary to amplify their outputs, rectify them and get the absolute difference between the two signals. The LVDT drive and signal detection circuit is shown in figure 3 . For this purpose, signals from the two secondaries are brought to two difference amplifiers, followed by precision rectifiers. The rectifier outputs are in turn given to a difference amplifier. Thus, the DC value of the absolute difference between the two signals is available for measurement.

The signal obtained from this amplifier against the displacement of the core is measured for linearity. For this purpose, the core is mounted on a micrometer and moved inside the LVDT and the signal obtained is measured by a digital multimeter. Readings are taken for a total displacement of $5 \mathrm{~mm}$. By adjusting the gains of intermediate amplifiers, the resolution achieved by this circuit is $1 \mathrm{mV} / \mu \mathrm{m}$.

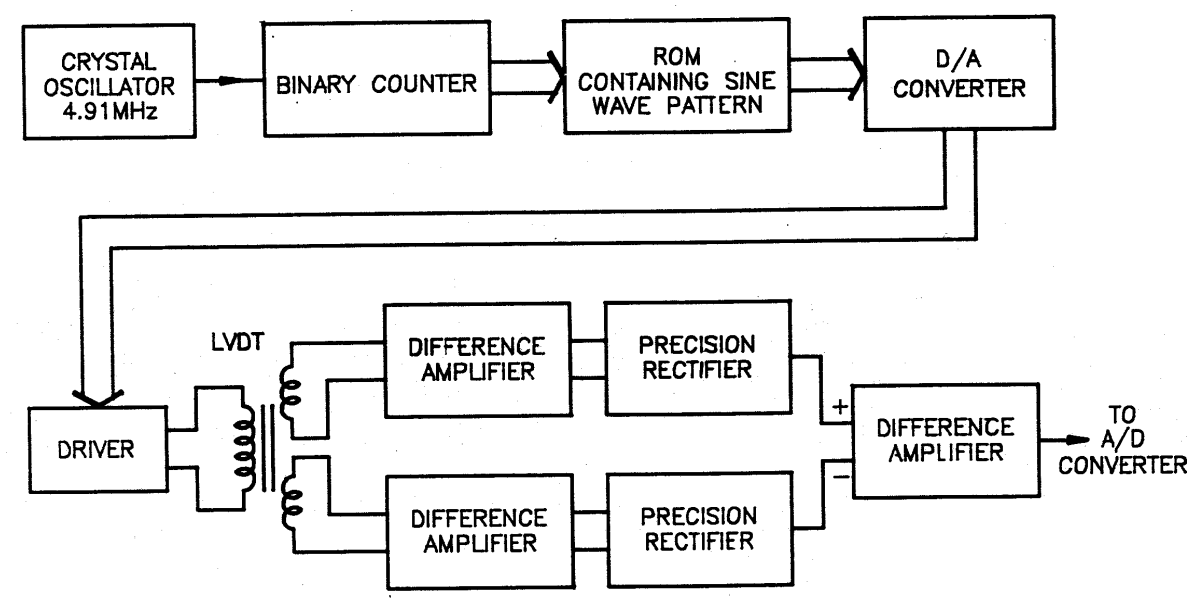

Figure 3. LVDT driver and measurement circuit. 
In order to measure the surface pressure, it is necessary to measure the force required to deflect the spring to which Wilhelmy plate is attached. For this purpose, the spring and LVDT are kept in air. The LVDT reading is kept in the middle of the scale by positioning of the core. A known set of weights are used to pull the spring and corresponding changes in the LVDT output are noted. The same procedure is carried out for two positions of the core inside the LVDT. It is seen that the maximum weight of $100 \mathrm{mg}$ causes a deflection of $1410 \mathrm{mV}$, or the deflection factor is $14.1 \mathrm{mV} / \mathrm{mg}$. This factor can be used for calculation of surface pressure.

2.2c A/D converter interface: For measurement of signal generated from the LVDT interface, we have chosen the ICL 7109 A/D converter. This A/D converter is of the dual slope type, has a resolution of 12 bits and an analog input range from -2.0 to +2.0 volts. The conversion time of this converter is $60 \mathrm{~ms}$ for a clock frequency of $6 \mathrm{MHz}$.

The digital interface of the A/D converter is provided through parallel $\mathrm{I} / \mathrm{O}$ ports of 8255. The ports PA and PB are programmed as input and used to read the 12 bits of the A/D converter, polarity and status bit. The analog input from the LVDT is brought to a buffer amplifier and fed to the A/D converter.

Control of the A/D converter is carried out with the help of the $\mathrm{R} / \mathrm{H}$ input, which is driven by PC4. For this purpose, this input is generally held low, maintains the A/D converter on hold or idle mode. Conversion is initiated by taking first $\mathrm{R} / \mathrm{H}$ input high. After this, the status but is checked and when it is low, conversion is complete. The 12bit data and polarity are then read by the microcomputer.

\subsection{The microcomputer system}

The control of various subsystems described so far is carried out by a microcomputer system, shown in figure 4 . The central processing unit is 8085 , chosen mainly because of its simple and useful architecture, availability, good software and hardware support etc. The system has $8 \mathrm{kB}$ EPROM and $8 \mathrm{kB}$ RAM. The EPROM the control program of LB film deposition set-up. A counter chip 8523 is included in the system for timing applications. Second channel of this counter chip is used by the LB films control program to generate interrupt RST6.5 at every $1 \mathrm{~ms}$. For communication with the personal computer, serial I/O port 8251 is provided. The serial data is sent and received through RS232 interface at 9600 baud. There are 3 parallel I/O ports included in the system, out of which two are used for driving the stepper motors and one is used for interfacing the A/D converter. The microprocessor is connected to the PC through COM port.

A control program is developed on PC to facilitate all possible operations likely to be carried out by the user. This includes operation of the setup manually in open or closed loop control, plotting of pressure-area isotherm for given monolayer and carrying out deposition of multiple layers automatically. In the open-loop mode, the substrate and/or the barrier motors are driven at appropriate speeds and directions. Alternately, if the closed-loop mode is required, a feedback mode bit has to be set. In this case, the substrate moves at the specified speed as before, but the barriers move only to bring the surface pressure back to initial level. 


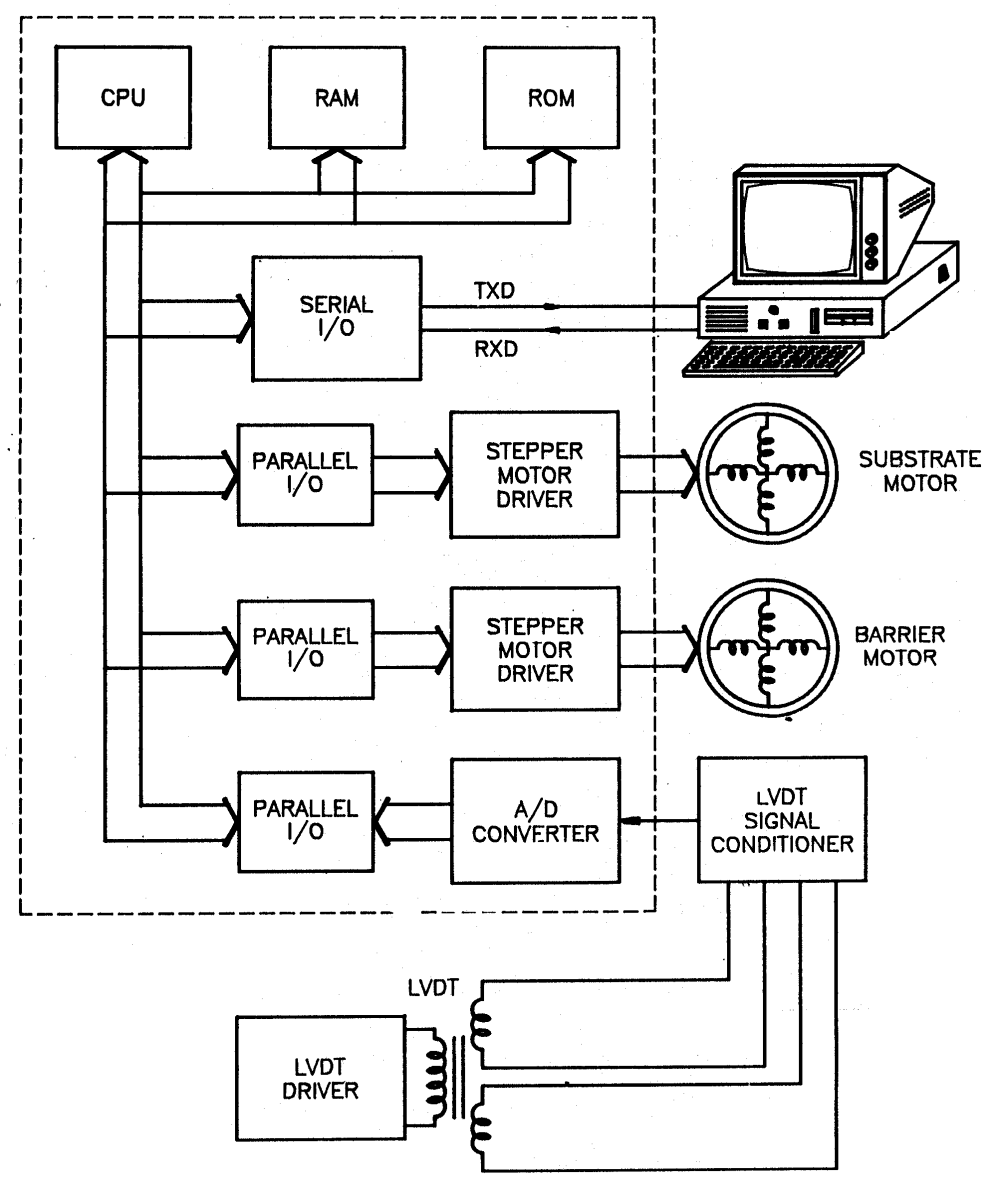

Figure 4. Block diagram of the control system.

This program is menu-driven, which allows the user to select various modes of operation through user-friendly commands. The program has following main features.

(1) Initial settings: These parameters include multiplication factors $(K)$ and offset $(O)$, each for substrate, barrier and LVDT, which are used for the calibration of the displacement and surface pressure. Normally, the motion of motors is displayed in steps. This can be modified for display in any suitable scale such as millimetres or microns. Similarly, the LVDT output in millivolts can be converted to read the same in millinewtons per metre.

(2) Manual operation of the substrate and the barriers: In this mode, various commands for the movement of the substrate and barrier motors are displayed on the screen. They are as follows.

Mode of operation: Preset (To run preset number of steps) or Continuous (To run motor continuously); Increment for barrier and substrate drives; Speed of barrier and substrate drives; Feedback on/off. 
The user can adjust the speeds of both motors and operate them in a single step, continuously or for a preset number of steps. The speeds and directions of the motor can be controlled on line also. This mode is suitable for the initial adjustments or for trials.

(3) Plot isotherm: This mode is suitable for plotting the isotherm of a given compound for its characterization or for compressing the monolayer before film deposition. Here, the user has to program the maximum displacement of barriers and speed. Then, as the Go command is given, the program provides an on-line plot of the surface pressure versus displacement, both in forward and reverse directions. These data can also be saved in a file and printed out.

(4) File execution mode: For the deposition of multiple layers, it is necessary to move the slider up and down number of times, with some time interval between consecutive depositions. The file execution mode is specially designed to provide this type of operation. For this, the commands available in the manual mode are written in a text file in a standard format. In addition, there are commands for loop operation, handshaking etc. Using these commands, a simple program can be written to carry out multiple depositions. In file execution mode, the program scans this file and carries out the commands sequentially, resulting in the deposition of the desired film.

As an example, consider a film with 10 layers, with 5 layers in up and 5 layers in down movement. A logical program can be written as follows.

$\begin{array}{lll}\text { S1 } & 100 & ; \text { Substrate speed }=100 \text { steps/second } \\ \text { S2 } & 500 & ; \text { Barrier speed = 500 steps/second } \\ \text { F } & 1 & ; \text { Feedback on } \\ \text { D } & 5 & \text {; Loop 5 times till Continue } \\ \text { X1 } & +10000 & \text {; Substrate increment in up direction = 10000 steps } \\ \text { G } & & ; \text { Go } \\ \text { L } & 10 & \text {; Wait for 10 seconds } \\ \text { X1 } & -10000 & \text {; Substrate increment in down direction = 10000 steps } \\ \text { G } & & \text {; Go } \\ \text { L } & 10 & \text {; Wait for 10 seconds } \\ \text { C } & & \text {; Continue } \\ \text { E } & & \text {; End. }\end{array}$

Thus the user can write program as per his requirements and the entire deposition process can be carried out automatically.

\section{Plot of pressure-area $(\pi-A)$ isotherm}

Monolayers of ferric stearate and zinc arachidate, known LB film forming compounds have been prepared at the air/water interface and their pressure-area isotherms were recorded by above LB set-up in order to check the performance of the set-up. For this purpose, chloroform solutions of millimolar concentration of the above compounds were separately spread on the water subphase of the trough. The water used for the 


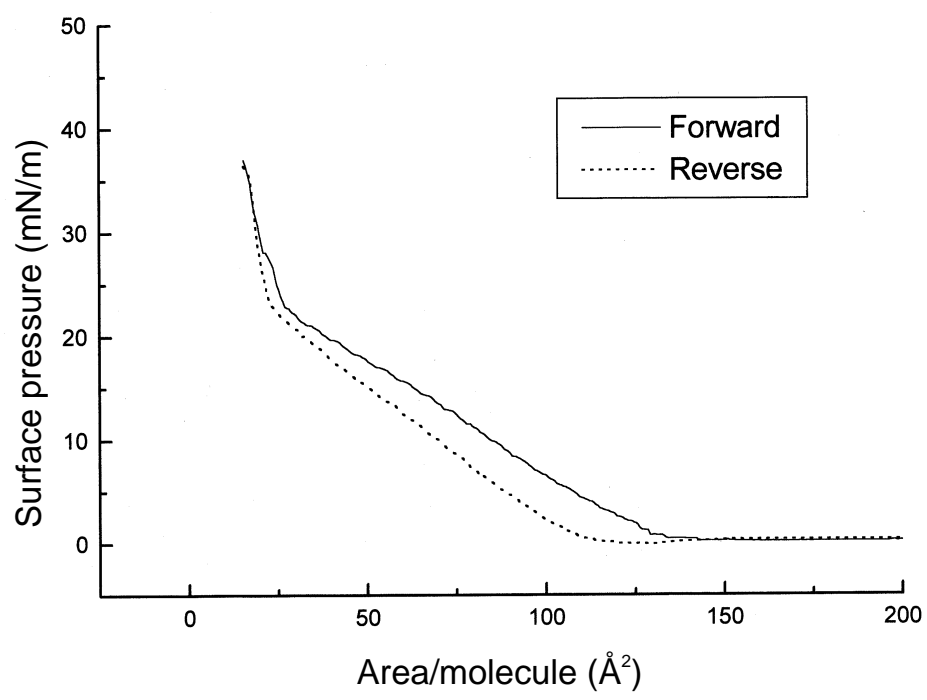

Figure 5. Plot of pressure-area isotherm of zinc arachidate.

subphase was Millipore-Q grade with a resistivity higher than $18 \mathrm{M} \Omega \mathrm{cm}$. Monolayers were compressed by moving the barriers in the forward direction till a sharp change in the displacement of the Wilhelmy plate, i.e., a sharp increase in the surface pressure was obtained. Then the barriers were moved backwards till the surface pressure lowers down to its initial value. The speed of barrier was kept $2.0 \mathrm{~mm} / \mathrm{min}$ both during the forward and the reverse motion. An on-line plot of this experiment was obtained which shows displacement of barriers versus LVDT output, as shown in figures 5 and 6 .

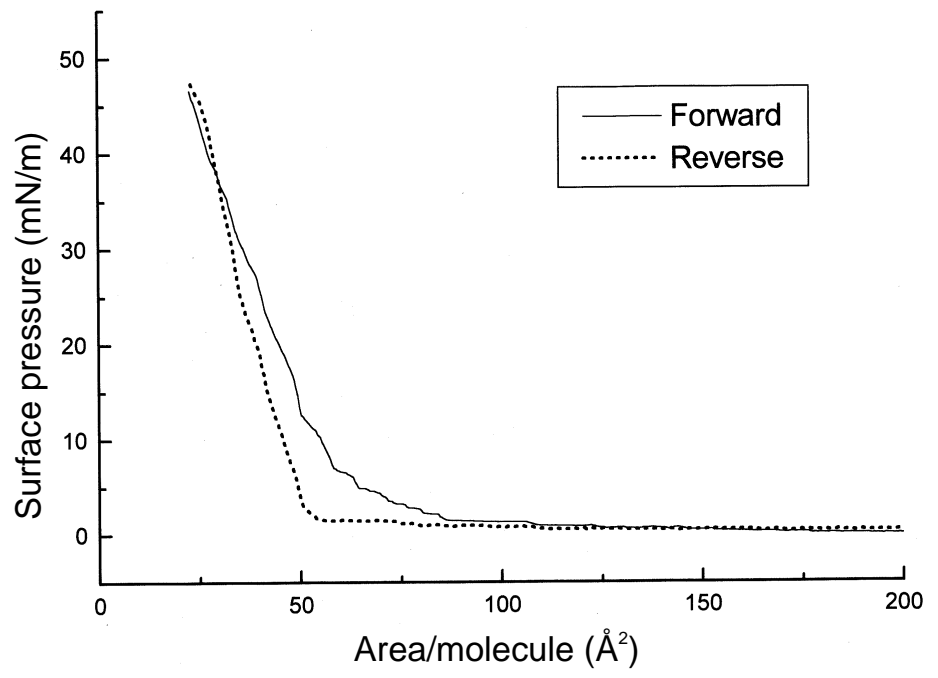

Figure 6. Plot of pressure-area isotherm of ferric stearate. 
Each of these figures represents the $(\pi-A)$ curves obtained during compression (i.e. forward motion of the barriers) and decompression (i.e. reverse motion of the barriers) of the monolayers. The shapes of these isotherms match quite well with the reported isotherms (Prakash et al 1987; Seidl et al 1999). The $\pi-A$ isotherm of zinc arachidate (figure 5) clearly shows three distinguished regions, viz. gaseous, liquid condensed and solid region. Whereas the $\pi-A$ isotherm of ferric stearate (figure 6) shows a relatively sharper increase in the surface pressure as compared to that of zinc arachidate, on compression after the gaseous region, i.e. at $\pi=\sim 5 \mathrm{mN} / \mathrm{m}$, leading to a very little or negligible liquid condensed region.

On decompression, the isotherm falls sharply to the $\pi=\sim 20 \mathrm{mN} / \mathrm{m}$ in case of zinc arachidate and from this point it moves somewhat slowly to the lower pressure and final transition to low pressure, i.e. gaseous region occurs at $\pi=\sim 3 \mathrm{mN} / \mathrm{m}$. The decompression curve of ferric stearate, on the other hand exhibits steep fall at $\pi=\sim 10 \mathrm{mN} / \mathrm{m}$, followed by slower decrease in pressure, till the final transition point which occurs in this case at $\pi=\sim 1 \mathrm{mN} / \mathrm{m}$.

Set of compression-decompression experiments have been carried out in both the above cases in order to explain the compression-decompression loop shown in the $\pi-A$ isotherm. We have found that the $\pi-A$ isotherm curves obtained on compressing the monolayer second time are identical with those obtained on first compression of the monolayer. This result indicates that the appearance of the area within the compression and decompression loop is not due to the collapse or nucleation of the monolayer. Rather, this indicates that the decompression process is basically different from the process during compression.

Generally, pressure-area isotherm is a plot of surface pressure versus area/molecule, for a given compound. In order to obtain the pressure-area isotherm plot in its original form, we have provided for the introduction of scaling factor and offsets for both axes in the program, so that $x$-axis is converted from displacement to area/molecule and $y$ axis is converted from LVDT output to surface pressure. However, it is not absolutely essential to enter these factors for LB film deposition, as the shape of the curve remains unchanged and one can choose and feed the optimum surface pressure and/or area of the monolayer in its original unit for the deposition of films.

\section{Deposition of LB films}

Fifteen-layered LB films of ferric stearate were deposited onto glass substrates. The target surface pressure for the deposition was selected from the solid state region of the $\pi-A$ isotherm. The monolayer was compressed up to the target pressure, then the deposition of the monolayer onto the glass substrate was done using file execution mode. Dipping speed was set to be $1 \mathrm{~mm} / \mathrm{min}$ for both upward and downward motions. By observing the meniscus of the subphase surface and the decrease in the distance between the barriers during deposition, it was concluded that the transfer of the monolayer from the water surface to the solid substrate occurred during both motions, i.e. Y-type deposition was obtained.

These films were characterised by low angle X-ray diffraction (XRD) analyses. Figure 7 shows $X$-ray diffraction pattern from a film made of 15 layers of ferric stearate on a glass substrate. Many orders of Bragg's peaks are clearly seen in this figure giving 


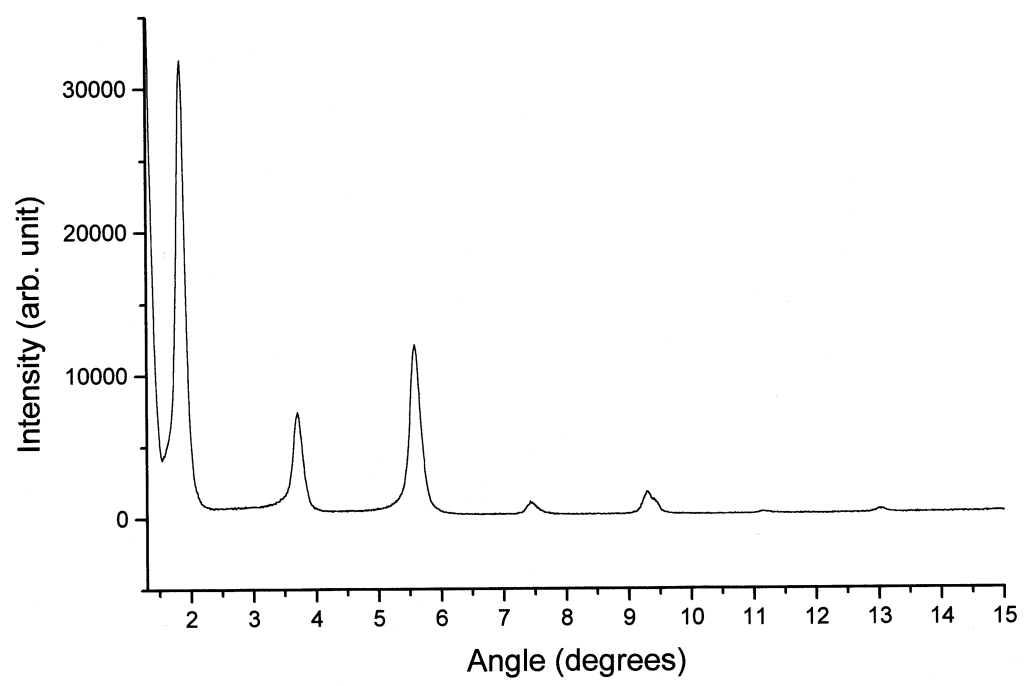

Figure 7. X-ray diffraction pattern of ferric stearate film.

information about the organization of the layers in the LB film. Several examples of such XRD patterns generated from different LB films are available in the literature (Bryce \& Petty 1995). Efforts are being made to obtain several structural as well as compositional information from these XRD spectra. From $d$-spacing, one can calculate the thickness of bilayer spacing as $\sim 47 \AA$ which is compatible with Y-type LB deposition.

\section{Conclusion}

A simple setup for the deposition of LB films is described. It includes hardware for the movement of the substrate and the barriers based on stepper motors, and the measurement of the surface pressure based on the Wilhelmy plate. The system is controlled by a computer, which runs a menu-driven software for its operation. Using this system, pressure-area isotherms of various organic compounds have been plotted and their films have been deposited successfully.

We would like to thank Drs K C Rustogi and Ajay Gupta for their keen interest and encouragement in the development of this setup. We would also like to thank N R Biswas and Rajendra Singh for wiring the circuits.

\section{References}

Albrecht O 1983 The construction of a microprocessor controlled film balance for precision measurements of isotherms and isobars. Thin Solid Films 99: 227-234 
Benming Ch, Deilacher F, Hoch M, Keller H J, Peiji W, Garnter S, Kahlich S, Schweitzer D 1991 Organic metals from chiral bedt-ttf donors. Lower Dimensional System and Molecular Electronics, NATO Asi Series B. 248 (eds R M Metzger, P Day, G C Papavassiliou (New York: Plenum) pp 175-180

Brockman H L, Jones C M, Schwebke C J, Smaby J M, Jarvis D E 1980 Application of a microcomputer film balance system for collection and analysis of data from mixed monolayers. J Colloid Interface Sci 78: 502

Bryce M R, Petty M C 1995 Electrically conductive Langmuir-Blodgett films of charge-transfer materials. Nature (London) 374: 771-776

Casy, V, McMonagle J B 1996 Minority-carriers MIS tunnel diode hydrogen sensors. Sensors Actuators B30: 233-240

Ciscato D, Fehl A, Turolla L 1988 Microstep control of floppy disk drive. IEEE Transactions on Industrial Electronics 35: 572-575

Hann R A 1990 Molecular structure and monolayer properties. Langmuir-Blodgett films (ed.) G Roberts (New York: Plenum) pp 17-83

Kuhn H, Mobius D, Bucher H 1972 Spectroscopy of monolayer assemblies. Techniques in chemistry (eds) A Weissberger, B W Rossiter (New York: Wiley) vol. 1, part IIIB, pp 577702

Miller L S, Hookes D E, Travers P J, Murphy A P 1988 A new type of Langmuir-Blodgett trough. J. Phy. E21: 163-167

Navathe C P, Roy U N, Dashora B L, Singh R, Maheswari S, Kukreja L M 1998 A control system for Langmuir-Blodgett film deposition setup based on microstepping. Meas. Sci. Technol. 9: 540-541

Peterson I R 1985 A fully automated high performance LB trough. Thin Solid Films 134: 135141

Peterson I R 1992 Langmuir-Blodgett films. Molecular electronics (ed.) G J Ashwell (Taunton, UK: Research Studies Press) pp 117-206

Petty M C 1996 Film deposition. Langmuir-Blodgett films, an introduction (Cambridge: University Press) pp 39-64

Prakash M, Peng J B, Ketterson J B, Dutta P 1987 Deposition of LB films of ferric stearate. Thin Solid Films 146: L15-L17

Seidl M, Schurr M, Brugger A, Volz E, Voit E 1999 Zinc oxide thin films prepared by means of Langmuir-Blodgett multilayers. Appl. Phys. A68: 81-85

Tredgold R H 1994 Langmuir-Blodgett films. Order in thin films (Cambridge: University Press) pp 59-81

Ulman A 1991 Application of LB and SA films. Introduction to ultrathin organic films: From Langmuir-Blodgett to self assembly (Boston: Academic Press) pp 339-384

Wynne J 1990 Microstepping drive circuit for single supply systems. Application note, Analog Devices, Massachusetts USA 